Nervenarzt $2021 \cdot 92: 878-891$

https://doi.org/10.1007/s00115-021-01167-0

Angenommen: 28. Juni 2021

Online publiziert: 10 . August 2021

(c) Springer Medizin Verlag $\mathrm{GmbH}$, ein Teil von Springer Nature 2021

\section{Q}

\author{
Henrik Walter ${ }^{1,2} \cdot$ Anna Daniels ${ }^{1,2} \cdot$ Sarah A. Wellan ${ }^{1,2}$ \\ ${ }^{1}$ Klinik für Psychiatrie und Psychotherapie CCM, Forschungsbereich Mind and Brain, Charité - \\ Universitätsmedizin Berlin, corporate member of Freie Universität Berlin and Humboldt-Universität zu \\ Berlin, Berlin, Deutschland \\ ${ }^{2}$ Fakultät für Philosophie, Berlin School of Mind and Brain, Humboldt-Universität zu Berlin, Berlin, \\ Deutschland
}

\title{
Kognitive Neurowissenschaft des Positiven
}

\section{Positive Valenzsysteme der Research- Domain-Criteria-Initiative}

in voller Konsequenz umgesetzt wurde. Ein Grund für diesen neuen Ansatz war die Unzufriedenheit mit einer rein symptombasierten DSM-5-Klassifikation. $\mathrm{RDoC}$ wollte dagegen einen neuen, systematischen Forschungsrahmen für die Psychiatrie bereitstellen, in dessen Zentrum die Idee steht, psychische Störungen als gestörte Funktion neuronaler Schaltkreise („circuits") aufzufassen. Das Grundkonzept ist eine evidenzbasierte Systematik neurokognitiver Funktionen, von denen verlangt wurde, dass sie mit bekannten neuronalen Schaltkreisen in Verbindung gebracht werden können. Diese Domänen und ihre Subkonstrukte (vgl. den Einführungsartikel von Hirjak, Schwarz und Meyer-Lindenberg in dieser Ausgabe von Der Nervenarzt) sollen dann auf verschiedenen Ebenen („units of analysis"), vom Gen bis zum Selbstbericht, bei allen psychischen Störungen erforscht werden. Dies ist eine systematische Forschungsstrategie, die langfristig dazu führen könnte, entweder neue Krankheitsentitäten zu etablieren oder Gruppen von Erkrankungen neu $\mathrm{zu}$ stratifizieren (d.h. in Subgruppen $\mathrm{zu}$ differenzieren). Mit anderen Worten: Der RDoC-Ansatz ist psychiatrische Forschung als angewandte kognitive Neurowissenschaft [41].

In der ersten Version der RDoCMatrix wurden aufgrund von Konsensuskonferenzen mit Experten 23 verschiedene psychologische Konstrukte identifiziert, die in fünf verschiedene
Domänen („domains of functioning“) kategorisiert wurden [8]. Dies waren "negative Valenzsysteme“, „positive Valenzsysteme“ (PVS), „kognitive Systeme“, „Systeme für soziale Prozesse“ und „Erregungs-/modulatorische Systeme“. Das System ist von der Anlage her offen für Erweiterungen. So wurde tatsächlich kürzlich als sechste Domäne „sensomotorische Systeme“ [28] hinzugefügt und einige Konstrukte und Subkonstrukte wurden bearbeitet, geändert oder ergänzt. Hier werden wir uns auf positive Valenzsysteme fokussieren.

\section{Positive Valenzsysteme}

Bei positiven Valenzen (PVS) geht es nicht, wie der Laie vielleicht vermuten würde, um Glück, Liebe, Empathie, Bewunderung oder Verehrung (Themen der positiven Psychologie), sondern ausschließlich um Belohnungsverarbeitung. Dies hat zum einen damit zu tun, dass die Rolle von Verstärkern seit dem Behaviorismus sehr detailliert untersucht worden ist, zum anderen damit, dass die neuronalen Schaltkreise der Belohnungsverarbeitung im Tier und inzwischen auch im Menschen recht gut bekannt sind.

Wie schon erwähnt, entstand die initiale RDoC-Systematik aufgrund eines kontrollierten Prozesses, bei dem ausgewählte Experten neurokognitive Funktionen nach bestimmen Kriterien im Konsensverfahren zusammengestellt 
Hier steht eine Anzeige.

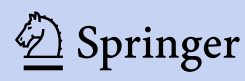


Tab. 1 Die Konstrukte der positiven Valenzsysteme der Research Domain Criteria in der früheren und aktuellen Version. (Mod. nach [29])

\section{Konstrukte/Subkonstrukte (2011)}

1. Annäherungsmotivation

1.1. Belohnungsbewertung

1.2. Anstrengungsbewertung/Bereitschaft zu arbeiten

1.3. Belohnungsantizipation/

Belohnungsvorhersagefehler

1.4. Handlungsauswahl/präferenzbasierte Entscheidungsfindung

2. Initiale Ansprechbarkeit auf Belohnung

3. Anhaltende Ansprechbarkeit auf Belohnung

4. Belohnungslernen

5. Gewohnheit

\section{Konstrukte/Subkonstrukte (2018)}

1. Ansprechbarkeit auf Belohnung

1.1 Belohnungsantizipation

1.2 Initiale Antwort auf Belohnung

1.3 Belohnungssättigung

2. Belohnungslernen

2.1. Probabilistisches Lernen/

Verstärkungslernen

2.2. Belohnungsvorhersagefehler

2.3. Gewohnheit

3. Belohnungsbewertung

3.1. Belohnung/Wahrscheinlichkeit

3.2. Verzögerung

3.3. Anstrengung hatten. Dieses Modell war von Anfang an ausdrücklich offen für wissenschaftlich begründete Veränderungen. Die PVSDomäne ist dafür ein gutes Beispiel. In der ersten Version 2011 [27] enthielten die PVS lediglich fünf Konstrukte, nämlich „Annäherungsmotivation“, „,initiale Ansprechbarkeit auf Belohnung “", , anhaltende Ansprechbarkeit auf Belohnung“, „Belohnungslernen“ und „Gewohnheit“. Doch schon bald wurde aufgrund der Vielzahl von Forschungsbefunden in diesen Bereichen klar, dass weitere Konstrukte Berücksichtigung finden sollten. In der Revision von 2018 (vgl. dazu [29]) kam es unter Einbeziehung weiterer Experten und nach transparenten Kriterien $\mathrm{zu}$ einer Erweiterung und Umordnung der PVS. Wichtig waren hier insbesondere die differenziertere Aufgliederung des Belohnungslernens mit Einschluss des Belohnungsvorhersagefehlers und die Überarbeitung des Konstrukts der anhaltenden Ansprechbarkeit auf Belohnung zum Subkonstrukt der Sättigung. Aktuell führt das RDoC drei Konstrukte mit je drei Subkonstrukten auf (vgl. - Tab. 1). Diese lassen sich gut am Beispiel von Nahrung, einem primären Verstärker, verdeutlichen.

Zucker wirkt als Verstärker und - zumindest bei Nagern - sogar stärker als Kokain. Das erste PVS-Hauptkonstrukt ist die Ansprechbarkeit auf Belohnung („reward responsiveness"). Darunter fällt als erstes Subkonstrukt die Erwartung („anticipation“) - z.B. das sprichwörtliche Wasser, das einem beim Rascheln des Schokoladenpapiers im Munde zusammenläuft. Das zweite Subkonstrukt ist die initiale Antwort auf die Belohnung („initial response to reward“), d.h. alles, was passiert, wenn eine Belohnung konsumiert wird, im Beispiel: all das, was in unserem Körper, Gehirn, Verhalten und subjektivem Erleben abläuft, wenn wir in einem hungrigen Zustand das erste Stück Schokolade essen. Das dritte Subkonstrukt ist der Prozess der Sättigung („satiation“). Das, was geschieht, wenn man genug Schokolade gegessen hat und das Bedürfnis danach sinkt oder sich gar in Widerwillen verwandelt.

Das zweite PVS-Hauptkonstrukt ist das Belohnungslernen („reward learning“): Wo kann man Nahrung finden? Wie lernt man die Wege dorthin, oder, um im Beispiel zu bleiben, wie kommt man am ehesten an Schokolade? Darunter fällt erstens probabilistisches und Verstärkungslernen („probabilistic and reinforcement learning“), also der jeweils positive Teil von Versuch und Irrtum, Belohnung und Bestrafung in der Dressur und umgangssprachlich auch als „Zuckerbrot und Peitsche“ („stick and carrot") bekannt. Es ist nicht nur das zentrale Element des Lernens bei Tieren, sondern bestimmt auch, ob uns das gefällt oder nicht, einen Großteil menschlichen Verhaltens. Zweitens fällt darunter der Belohnungsvorhersagefehler („reward prediction error"). Das neuronale Belohnungssystem kodiert nämlich nicht nur die Erwartung und den Erhalt von Belohnung, sondern repräsentiert auch deren Differenz, den „Fehler“; präziser, die Abweichung der tatsächlichen Belohnung von deren Vorhersage, d.h. ob etwas besser oder schlechter als erwar- tet ist, was sich analog zum invasiven Tierexperiment beim Menschen sehr gut mit der funktionellen Bildgebung zeigen lässt. Und drittens fallen unter Belohnungslernen die Gewohnheiten („habits“): Wenn ein Organismus etwas erlernt hat, wird es zunehmend automatisch durchgeführt. Der initial als sehr belohnend empfundene Konsum geht dabei nicht mehr unbedingt mit positiven Gefühlen einher: Man nascht, raucht, trinkt und verhält sich vielleicht nur noch aus Gewohnheit [12]. Neurobiologisch wird dies mit einer Verlagerung der Reaktionen im Belohnungssystem vom ventralen (mesolimbischen) zum dorsalen (motorischen) Striatum in Verbindung gebracht („ventral-to-dorsal shift").

Als drittes Hauptkonstrukt listet die RDoC-Matrix unter den PVS schließlich die Bewertung von Belohnung („reward valuation") auf. Die Bewertung von Belohnung hängt erstens davon $\mathrm{ab}$, wie groß und wie wahrscheinlich die Belohnung ist („reward/reward probability“), zweitens, wie lange es dauert, bis man sie erhält („delay“ - in der Regel werden spätere Belohnungen abgewertet $=$ „temporal discounting“) und zeigt sich schließlich drittens darin, wie viel Anstrengung („,effort“) ein Organismus bereit ist aufzuwenden, um eine mögliche Belohnung zu erhalten [29].

\section{》) Bei neurokognitiven \\ PVS-Systemen spielt das mesolimbische Dopaminsystem eine zentrale Rolle}

All diese kognitiven Funktionen sind sowohl im Tierexperiment als auch beim Menschen sehr gut untersucht, was letztendlich der Grund dafür ist, dass sie in die RDoC-Matrix aufgenommen wurden. Bei fast allen neurokognitiven PVS-Systemen und Grundfunktionen spielt das mesolimbische Dopaminsystem (vgl. - Abb. 1) eine zentrale Rolle. Diese Grundfunktionen wurden schon lange vor der RDoC-Initiative, teils seit Jahrzehnten im Bereich dreier großer psychischer Störungen untersucht, nämlich der Sucht $[6,17,34]$, der Schizo- 
Hier steht eine Anzeige.

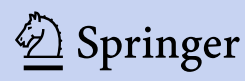


phrenie $[11,14,24]$ und der Depression $[5,14,21,24,38,43]$. In der Sucht beispielsweise spielen am Beginn die initiale Ansprechbarkeit auf Drogen sowie die verminderte Fähigkeit zum Belohnungsaufschub (Verzögerung) wesentliche Rollen. Im weiteren Verlauf werden durch Prozesse des Belohnungslernens Verhaltensmuster etabliert, die dann den Drogenkonsum zur Gewohnheit werden lassen. Im Bereich der Schizophrenie wurde insbesondere der Vorhersagefehler bei der Wahnentstehung untersucht $[7,11]$. Und bei der Depression ist die These der verminderten positiven Verstärker schon seit Jahrzehnten eine Säule des Depressionsverständnis und im Bereich der Behavioral-ActivationTherapie [20] auch therapeutisch relevant (vgl. dazu auch die Anmerkungen weiter unten bei der Anhedonie).

Man könnte nunmehr versuchen, Untersuchungen $\mathrm{zu}$ allen oder zumindest $\mathrm{zu}$ einer dieser großen Erkrankungen aus RDoC-Perspektive zusammenzufassen. Doch erstens würde dies aufgrund der überwältigenden Vielzahl an Untersuchungen in einem Artikel gar nicht möglich sein oder aufgrund der Vielzahl der Konstrukte zu einer letztlich willkürlichen Auswahl führen. Zweitens gibt es schon sehr viele und exzellente Übersichtartikel zur Rolle des Belohnungssystems bei diesen Erkrankungen. Und drittens fällt auf, dass sich keine größeren Theorieartikel zu diesen Erkrankungen aus RDoC-Perspektive finden lassen. Dies legt nahe, dass der RDoC-Ansatz hier keinen entscheidenden Impuls zu neuen Forschungen oder Ansätzen bewirkt, sondern eher vorhandenes Wissen systematisiert hat. Daher wurde in diesem Artikel ein anderes Vorgehen gewählt. Zum einen haben wir einen transdiagnostischen Ansatz, eine Stärke von RDoC, gewählt und betrachten das Phänomen der Anhedonie aus RDoC-Perspektive. Zweitens werden wir durch Darstellung zweier rezenter Metaanalysen zeigen, dass auch ganz andere Erkrankungen, hier die Autismusspektrumstörungen und die Gruppe der Essstörungen (Essen ist ein primärer Verstärker), durch eine RDoCPerspektive einen neuen theoretischen Blick erlauben und die Entwicklungsper-

Nervenarzt 2021 · 92:878-891 https://doi.org/10.1007/s00115-021-01167-0

(c) Springer Medizin Verlag GmbH, ein Teil von Springer Nature 2021

\author{
H. Walter · A. Daniels · S. A. Wellan
}

\title{
Kognitive Neurowissenschaft des Positiven. Positive Valenzsysteme der Research-Domain-Criteria-Initiative
}

\section{Zusammenfassung}

In diesem Artikel werden die Domäne "positive Valenzsysteme" (PVS) der Research-Domain-Criteria(RDoC)-Matrix sowie ihre Subkonstrukte dargestellt und erläutert. Unter PVS fallen im Wesentlichen verschiedene Formen und Prozesse der Belohnungsverarbeitung. Diese werden in der Psychiatrie schon seit Jahrzehnten im Bereich von Sucht, Schizophrenie und Depression untersucht und letztere sind daher nicht Gegenstand dieses Artikels. Hier soll vielmehr die heuristische Fruchtbarkeit der RDoCSystematik für das Verständnis anderer Erkrankungen und Konstrukte dargestellt werden und zwar für das transdiagnostische

Konstrukt der Anhedonie sowie für die Autismusspektrumstörung und die Gruppe der Essstörungen. Weiterhin wird gezeigt, wie die PVS-Domäne auch klinisch den Blick über die traditionelle Psychopathologie erweitert und wie sie die Entwicklung neuer behavioraler Messinstrumente angeregt hat. Abschließend wird auf Limitationen und mögliche zukünftige Erweiterungen des Ansatzes eingegangen.

Schlüsselwörter

Belohnungsverarbeitung · Anhedonie . Essstörungen · Autismusspektrumstörung · Selbstbericht

\section{Positive cognitive neuroscience. Positive valence systems of the Research Domain Criteria initiative}

Abstract

In this paper, the domain positive valence systems (PVS) of the Research Domain Criteria (RDoC) matrix and its subconstructs are presented and discussed. The PVS basically reflect different forms and aspects of reward processing. These have been investigated in psychiatry in the context of addiction, schizophrenia and depression for decades; the latter are therefore not the topic of this paper. This article presents the heuristic value of the RDoC system in understanding other disorders and constructs, namely the transdiagnostic symptom of anhedonia, autism spectrum disorder and eating disorders. In addition, it outlines how the PVS domain has also enriched the clinical perspective of traditional psychopathology and stimulated the development of new behavioral measurement instruments. Finally, the limitations and potential future developments of the framework are discussed.

\section{Keywords}

Reward processing · Anhedonia · Eating disorders · Autism spectrum disorder · Selfreport instruments spektive betonen (so ähnlich wie oben bei der Sucht kurz geschildert). Und drittens wollen wir ein Hauptargument gegen das RDoC, nämlich dass es als Forschungssystematik ganz interessant sein mag, klinisch jedoch unbrauchbar sei, entkräften, indem wir zeigen, wie es ganz konkret in die Klinik übertragen werden kann und wie es u. a. dazu geführt hat, ein neues, PVS-spezifisches Instrument zu entwickeln.

\section{Anhedonie}

Der Begriff der Anhedonie, die Unfähigkeit Lust und Freude zu empfinden, wurde 1896 durch den französischen Psychologen Theodule Ribot in Analogie zur Analgesie - der Unfähigkeit, Schmerz zu empfinden - in die Literatur eingeführt [30]. Genau das versteht auch der klinisch tätige Psychiater darunter. Anhedonie ist eines der Hauptsymptome der Depression nach DSM-5 und der International Statistical Classification of Diseases and Related Health Problems 10 (ICD-10), aber auch ein wichtiges Symptom der Schizophrenie und bei Abhängigkeitserkrankungen; darüber hinaus tritt sie jedoch auch bei einer großen Anzahl anderer psychischer Störungen auf und ist 


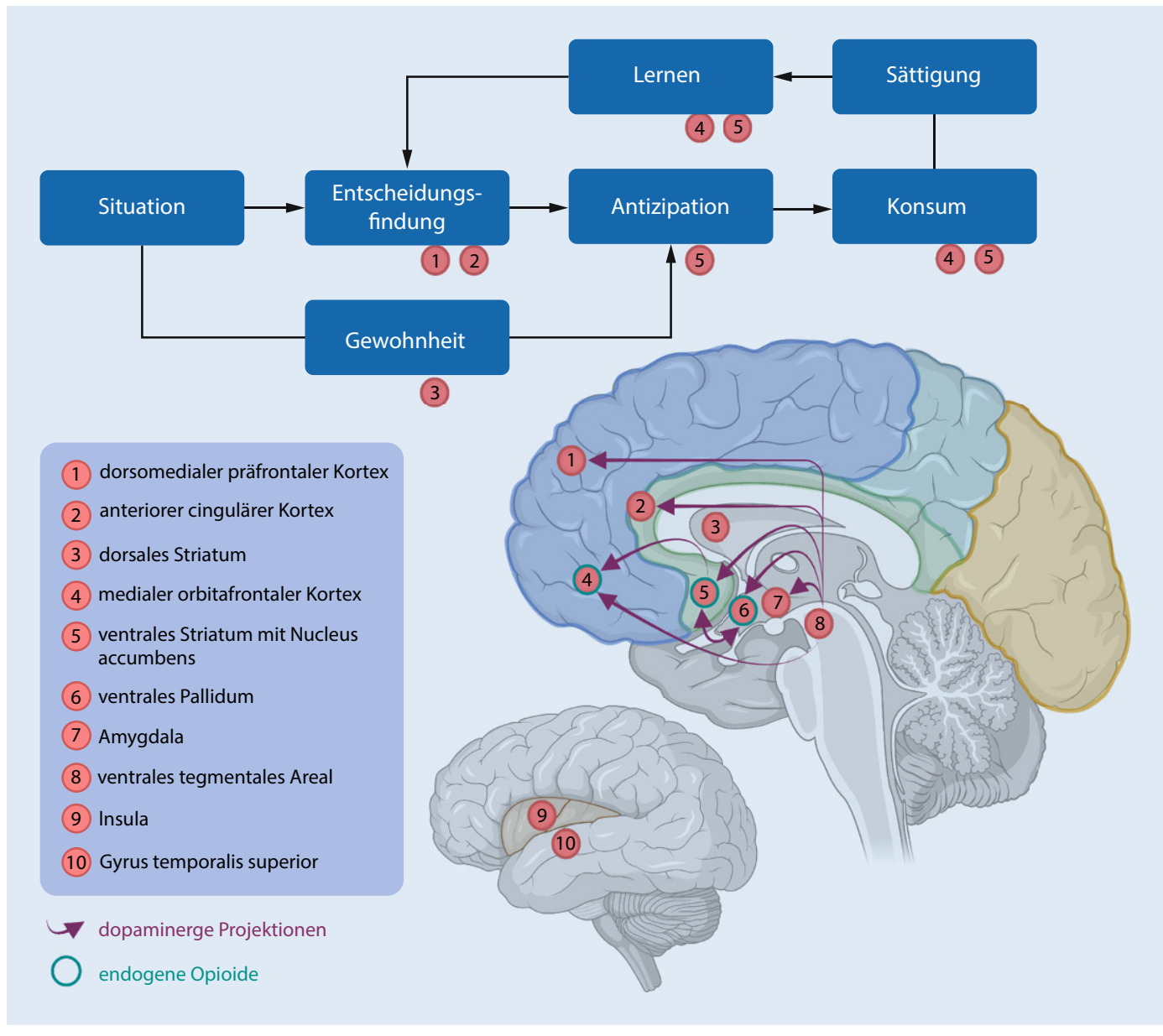

Abb. $1 \varangle$ Vereinfachtes Modell der (an-)hedonischen Teilprozesse und dafür relevanter neuronaler Mechanismen, darunter dopaminerge Projektionen vom ventralen tegmentalen Areal zum ventralen Striatum mit Nucleus accumbens. Regionen im Striatum projizieren zu unterschiedlichen Bereichen des medialen präfrontalen Kortex, u. a. zum medialen orbitofrontalen Kortex, zum dorsomedialen präfrontalen Kortex und zum anterioren zingulären Kortex. Endogene Opioide spielen v. a. beim Konsum bzw. bei der initialen Reaktion auf Belohnung eine Rolle, indem sie das hedonische Erleben bei Belohnungserhalt vermitteln. (Mod. nach [42]. Bilder Hirn: mit freundlicher Genehmigung, $\odot$ BioRender.com, alle Rechte vorbehalten)

auch bei manchen Personen ohne psychiatrische Diagnose zu finden [39].

\section{》) Anhedonie ist ein modularer Prozess der gestörten Belohnungsverarbeitung}

Doch tatsächlich geht es bei der Anhedonie um mehr. Schon in gängigen Depressionsfragebögen hat die Anhedonie zwei Aspekte, neben dem Verlust von Freude auch den Verlust des Interesses an normalerweise freudvollen Tätigkeiten, also eine motivationale Komponente. Vielleicht wegen dieser Betonung des Verlustes wird die Anhedonie in der RDoC-Matrix übrigens noch, aus unserer Sicht fälschlicherweise, unter dem Subkonstrukt Verlust („loss") der negativen Valenzsysteme aufgeführt. Dabei macht das Studium der Anhedonieforschung mehr als deutlich, dass Anhedonie heutzutage als ein modularer Prozess der gestörten Belohnungsverarbeitung mit verschiedenen Teilpro- zessen angesehen wird, die sich direkt auf die PVS der RDoC-Matrix beziehen. (Für eine zusammenfassende deutschsprachige Darstellung vgl. [42], für rezente englischsprachige Übersichtsarbeiten vgl. $[10,18,43])$.

\section{》) Der Genuss beim Konsum wird vor allem durch endogene Opioide vermittelt}

In Abb. 1 wird der zeitliche Ablauf der Informationsverarbeitung verdeutlicht, der in einem Organismus bei der Konfrontation mit einer möglichen Belohnung stattfindet, und die jeweils wichtigsten Hirnregionen dafür werden dargestellt. Als Erstes muss der Organismus abwägen und entscheiden, ob es sich lohnt, sich um diese Belohnung zu bemühen. Der damit einsetzende motivationale Prozess ist vor allem durch die Antizipation der potenziellen Belohnungsbefriedigung bestimmt. Beim
Erhalt bzw. Konsum der Belohnung entstehen in der Regel angenehme Gefühle. Ein Konsum wird in der Regel so lange fortgesetzt, bis eine Sättigung eintritt, deutlich und beispielhaft bei den primären Verstärkern Nahrung und Sexualität. In einem weiteren Schritt erfolgt dann eine automatische, beim Menschen manchmal auch reflektierte Bewertung, ob der ganze Aufwand der Mühe wert war, und der Organismus lernt daraus. Mit der Wiederholung belohnender Tätigkeiten werden sich dann Gewohnheiten entwickeln, um sich die kognitiv aufwendigen Abwägungsprozesse in ähnlichen Situationen zu ersparen - zum Besseren oder eben auch zum Schlechteren, wie der Genuss ungesunder Nahrung und Substanzen und die Ausübung langfristig schädlicher Aktivitäten zeigt. Modelle dieser Art liegen der Anhedonieforschung zugrunde und zeigen den Bezug zu den RDoC-PVS klar auf. 
Warum ist dies für klinische Belange relevant? Nun, eine zentrale Annahme der modernen Anhedonieforschung ist, dass eine Störung jedes dieser Teilprozesse das Symptom der Anhedonie verursachen oder zu dessen Erhalt beitragen kann. Entgegen gängigen Überzeugungen könnte etwa bei Depressiven die Fähigkeit zum Genuss von Belohnungen erhalten und möglicherweise eher das Interesse/die Erwartung [14,36] und/ oder das anstrengungsbasierte Entscheiden gestört sein $[5,38]$. Dies hat natürlich Implikationen für die Behandlung. Diese sind schon in der Verstärkertheorie der Depression von Lewinsohn [25] und heute in der Behavioral-Activation-Therapie [20] gesehen und umgesetzt worden: Dort gilt, einen anhedonen depressiven Patienten dazu zu bringen, etwas zu tun, um überhaupt erst die Möglichkeit zu eröffnen, positive Erfahrungen $\mathrm{zu}$ machen. Eine Erkenntnis aus tierexperimentellen Untersuchungen ist zudem, dass für die Erwartung und das Belohnungslernen das Dopamin eine zentrale Rolle spielt, während der eigentliche Genuss beim Konsum vor allem durch Opioide vermittelt wird - im normalen Kontext durch endogene Opioide, bei der Opioidabhängigkeit durch künstliche [4]. Die Rolle des Dopamins erklärt unter anderem die unerwünschten Nebenwirkungen antidopaminerger Substanzen wie klassischen Antipsychotika mit resultierender Amotivation und verminderter Lernfähigkeit.

Neuere klinische Fragebögen zur Anhedonie versuchen, die verschiedenen Komponenten auch im Selbstbericht zu erfassen (z.B. die TEPS - Temporal Experience of Pleasure Scale [13] oder die DARS - Dimensional Anhedonia Rating Scale [31]). Allerdings zeigt sich, dass die neurobiologisch differenzierbaren Komponenten im Selbsterleben nur selten getrennt werden können (mehr dazu im Abschnitt „Selbstbericht über PVS“).

\section{Essstörungen}

Ein naheliegendes Krankheitsbild, bei dem PVS eine Rolle spielen könnten, sind Essstörungen, da es hier um die krankhafte veränderte Aufnahme oder eben
Restriktion eines überlebensnotwendigen primären Verstärkers, nämlich Essen, geht. In einer rezenten Übersichtsarbeit untersuchen Schaefer und Steinglass daher Studien zu Belohnung und Essstörungen aus einer RDoC-Perspektive [32]. Sie fanden 75 jährliche Publikationen zum Stichwort „Essstörungen“ und „Belohnung“ zwischen 2010 und 2019, deutlich mehr als vor der Einführung des RDoC-Ansatzes im Jahr 2009. Häufig wurden Belohnungserwartung und -erhalt essensrelevanter oder körperlicher Stimuli untersucht. Schaefer und Steinglass konzentrieren sich hier aber auf ein anderes Konstrukt, nämlich das Belohnungslernen mit seinen drei Subkonstrukten probabilistisches Lernen und Verstärkungslernen, Belohnungsvorhersagefehler und Gewohnheit. Der Grund liegt in ihrer entwicklungspsychiatrischen Perspektive. Sie möchten verstehen, wie Individuen ihr Essverhalten im Laufe ihrer Entwicklung lernen. Sie analysieren dabei hauptsächlich Selbstberichte, psychologische Paradigmen sowie Untersuchungen mit bildgebenden Verfahren.

Zusammengefasst fanden Schaefer und Steinglass [32], dass probabilistisches Lernen und Verstärkungslernen häufiger untersucht wurden und es auch Hinweise auf Störungen bei Essstörungen in diesem Subkonstrukt gibt. Für den Vorhersagefehler gibt es eine Reihe an Bildgebungsstudien, jedoch kaum Befunde mit den in der RDoC-Matrix empfohlenen Paradigmen. Die Selbstberichtliteratur gibt Hinweise darauf, dass belohnungsbezogene Erwartungen in Bezug auf Essen und Schlankheit eine wichtige Rolle für den Beginn und die Aufrechterhaltung von Essstörungen spielen. Für Anorexia nervosa gibt es Hinweise aus der Selbstberichtliteratur, dass ungünstige Verhaltensroutinen mit Mechanismen von Gewohnheiten zu tun haben; für Störungen bei der Herausbildung von Gewohnheiten gibt es dagegen keine Hinweise. Schaefer und Steinglass [32] schließen, dass hier ein großer Forschungsbedarf besteht, und schlagen dazu ein entwicklungsorientiertes Modell zur Untersuchung von Essstörungen vor: Während probabilistisches und Verstärkungslernen v.a. in der initialen Symptomentwicklung von Bedeutung sein sollten (Lerntyp: Response-Outcome), sollten mit zunehmender Chronifizierung vor allem der Vorhersagefehler (Lerntyp: Erwartung eines Outcomes) und dann schließlich Gewohnheiten (Lerntyp: StimulusResponse) eine Rolle spielen. Dieser Ansatz ist ein gutes Beispiel dafür, wie heterogene Forschungsaktivitäten in ein Modell überführt werden können, bei dem RDoC-PVS-Konstrukte eine strukturierende Rolle spielen.

\section{Autismusspektrumstörung}

In einem ähnlichen Ansatz, also RDoCPVS als heuristischer Rahmen, Analyse der Literatur und Formulierung eines Entwicklungsmodells, untersuchten Tschida et al. Autismusspektrumstörungen [40]. Als Hintergrund dient die soziale Motivationshypothese des Autismus: Autistische Personen erleben soziale Aspekte aus ihrer Umwelt als weniger belohnend, also Freude bereitend, als neurotypische Individuen und entwickeln daher auch weniger soziale Fertigkeiten, was zu einem selbstverstärkenden Kreislauf führt. Neben den sozialen Belohnungen betonen neuere Ansätze auch die belohnende Wirkung von RRBIs („restricted and repetitive behaviors and interests"), also stereotypen, ritualisierten Verhaltensmustern und Interessen. Die Autoren identifizierten in einer präregistrierten Literatursuche nach den PRISMA(„preferred reporting items for systematic reviews and metaanalyses")-Richtlinien von initial 334 letztendlich 34 Arbeiten, die sie ausführlich evaluierten, ohne sie aber, wegen der geringen Anzahl pro (Sub-)Konstrukt, zu metaanalysieren. Sie führten aber ausführliche Detailanalysen der Ergebnisse pro (Sub-)Konstrukt durch, die hier zusammenfassend geschildert werden.

Insgesamt waren die Arbeiten von hoher Qualität. Im Bereich der Ansprechbarkeit auf Belohnung fanden sich Unterschiede zwischen Patienten- und Kontrollgruppen zumeist nur auf neuronaler, nicht auf Verhaltensebene. Unterschiede der neuronalen Aktivierung zum Zeitpunkt der initialen Ansprechbarkeit 
zeigten dabei einen potenziellen $\mathrm{Zu}$ sammenhang zu RRBIs, zum Zeitpunkt der Antizipation zu RRBIs und sozialen Defiziten. Während die Insula konsistent an der initialen Ansprechbarkeit beteiligt war, waren der Nucleus accumbens und die Amygdala eher in die Belohnungsantizipation involviert. Für das Subkonstrukt der Sättigung gab es keine Studien. Für Belohnungslernen zeigte sich ein potenzieller Zusammenhang sowohl von probabilistischem Lernen und Verstärkungslernen als auch von Vorhersagefehlern mit sozialen Defiziten, während zumindest eine Studie nahelegte, dass Gewohnheiten intakt waren. Auf neuronaler Ebene waren, über die Studien hinweg, der Nucleus accumbens, der Gyrus temporalis, der präfrontale sowie der orbitofrontale Kortex mit probabilistischem und Verstärkungslernen assoziiert. Für den Vorhersagefehler fanden sich aberrante Aktivierungen im Nucleus accumbens, jedoch nicht konsistent über Studien hinweg, und für Gewohnheiten gab es keine Bildgebungsstudien. Für die Belohnungsbewertung zeigten sich für alle drei Subkonstrukte (Wahrscheinlichkeit, Verzögerung, Anstrengung) potenzielle Zusammenhänge sowohl mit RBBIs als auch sozialen Defiziten. Neuronal zeigten sich für das Subkonstrukt Verzögerung vor allem Zusammenhänge mit präfrontalem Kortex, Kleinhirn und anteriorem zingulärem Kortex; Bildgebungsdaten zu Zusammenhängen mit Wahrscheinlichkeit oder Anstrengung lagen keine vor. Tschida et al. [40] verweisen - zu Recht - auf Limitationen ihrer Analyse, nämlich, die recht heterogene Datenlage, fehlende Untersuchungen zu manchen Subkonstrukten, die begrenzte Anzahl eingesetzter Belohnungstypen und der mögliche Einfluss übergeordneter kognitiver Funktionen wie Aufmerksamkeit, Exekutivfunktionen oder Arbeitsgedächtnis in einigen Studien.

Ähnlich wie Schäfer et al. für die Essstörungen formulieren Tschida et al. [40] dann ein Entwicklungsmodell, das sie als Weiterentwicklung des Imbalance-Modells von Kohls [23] ansehen. Dieses hatte eine generelle Imbalance in der Verarbeitung von Belohnungen postuliert, die dadurch erklärt wird, dass RRBIs kontinuierlich positiv verstärkt werden. Die Autoren ergänzen dies durch das Postulat der Relevanz spezifischer PVS-Konstrukte zu bestimmten Phasen der kindlichen Entwicklung: Während im Alter von 0 bis 12 Monaten vor allem eine verminderte Ansprechbarkeit auf Belohnung eine Rolle spiele (Symptomebene: verminderte Reaktion auf den eigenen Namen), würden im Alter von 12 bis 48 Monaten dann Probleme mit dem Belohnungslernen dazu kommen (Symptomebene: verminderter geteilter Affekt und Aufmerksamkeit, Auftauchen repetitiver Gewohnheiten, Bewegungs- und Spielverhaltens, Insistieren auf „Immerdas-Gleiche“). In der weiteren Entwicklung würden Defizite in der Ansprechbarkeit auf Belohnung und der Belohnungsbewertung dominieren (Symptome: Erfahrung und Lernen resultieren in einer veränderten Ansprechbarkeit auf soziale Situationen und $\mathrm{zu}$ einer Bevorzugung von Spezialinteressen gegenüber sozialem Engagement; [40]).

\section{I) Spezifische PVS-Konstrukte sind zu bestimmten Phasen der kindlichen Entwicklung relevant}

Die beiden Beispiele Essstörungen und Autismusspektrumstörungen zeigen sehr gut, was der RDoC-Ansatz eigentlich ist. Es ist kein Krankheitsmodell, es ist auch kein Klassifikationssystem, sondern es ist ein vom DSM-5 unabhängiger Rahmen, der es erlaubt, Forschung zu psychischen Störungen systematisch und auf der Grundlage neuer Erkenntnisse zu ordnen und Modelle zu entwickeln, die weitere Forschungen leiten können. Derzeit sind die Einflüsse der RDoC-PVSSystematik im Bereich der Autismusspektrumstörungen und der Essstörungen eher forschungs- als praxisrelevant.

\section{Translationale Aspekte („, bench- to-bedside“") der RDoC-PVS}

Einige häufig geäußerte Kritikpunkte an der RDoC-Initiative sind, dass sie $\mathrm{zu}$ reduktionistisch, d.h. zu neurowissenschaftlich orientiert sei, die subjektive Erfahrung des Patienten weitgehend ignoriere, für klinische Zwecke unbrauchbar sei und das DSM-System in der klinischen Praxis niemals ersetzen werde könne. Richtig daran ist, dass die Grundidee des RDoC-Ansatzes neurobiologisch inspiriert ist, da sie nur diejenigen psychologischen Konstrukte erlaubt, zu denen es Wissen über mindestens einen damit verbundenen neuronalen Schaltkreis („neural circuits") gibt. Und in der Tat: Innerhalb der RDoCMatrix gibt es keinen vorgesehenen Platz für klinische Symptome, nur subjektive Selbstberichte tauchen dort als höchstes Level der unterschiedlichen Ebenen auf, auf denen die Konstrukte untersucht werden sollen. Den Anspruch, das DSM-5-System in der Praxis zu ersetzen, hatten die Konstrukteure des $\mathrm{RDoC}$ allerdings nie erhoben. Der RDoC-Ansatz ist primär als ein systematischer Rahmen für die psychiatrische Forschung gedacht, die nicht durch DSM-5-Kategorien voreingenommen sein sollte. Doch inzwischen gibt es auch Anstrengungen, die Ebene subjektiver Berichte zu verbessern und für die klinische Praxis nutzbar zu machen.

\section{Selbstbericht über PVS: die Positive Valence Systems Scale}

Können die neurokognitionswissenschaftlich gut untersuchten psychologischen Konstrukte auch im subjektiven Erleben von Personen erfasst werden? Das typische Vorgehen dazu ist die Entwicklung und Validierung von Fragebögen. So haben Khazanov et al. eine Skala entwickelt, die Positive Valence Systems Scale (PVSS), die einfach auszufüllen, gut verständlich und alltagsrelevant ist [22]. Ausgangspunkt zur Entwicklung war die PVS-Systematik von 2011 (vgl. - Tab. 1). Die Autoren führten insgesamt drei Studien in gesunden und klinischen Stichproben durch und etablierten aus einer 45 Items umfassenden Langversion, die primäre und sekundäre Verstärker in verschiedenen Lebensbereichen erfasste, eine Kurzversion mit 21 Fragen (PVSS21). Beispiele dieser Fragen sind: „Ich freute mich auf das anstehende Essen“ (Belohnungstyp Essen, PVS-Konstrukt Belohnungsantizipation) oder „Es fühlte sich gut an, mit jemanden im körper- 
Hier steht eine Anzeige.

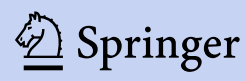


Hier steht eine Anzeige.

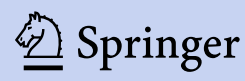


lichen Kontakt $\mathrm{zu}$ sein, dem ich mich nahe fühlte" (Belohnungstyp körperliche Berührung, PVS-Konstrukt initiale Ansprechbarkeit auf Belohnung) oder „Ich habe Energie in Aktivitäten investiert, die mir Freude bereiten" (Belohnungstyp Hobbys, PVS-Konstrukt Anstrengungsbewertung). Für eine vollständige Liste aller Items siehe Khazanov et al. [22]. Jede Frage ist auf einer 9-Punkte-Skala zu bewerten, die von „extrem zutreffend (im Original: „true“) für mich" bis hin $\mathrm{zu}$ „extrem unzutreffend („untrue“) für mich“ (Übersetzungen H.W.) geht, und zwar wegen der Relevanz der Fragen für die Depression, bezogen auf den Zeitraum der letzten zwei Wochen; es handelt sich also um einen State-Fragebogen.

\section{》) Die PVSS-21 zeigte eine hohe interne Konsistenz und Reliabilität}

Die finale PVSS-21 umfasst jeweils drei Fragen in sieben Subskalen von Belohnungstypen (Essen, körperliche Berührung, Outdooraktivitäten, positives Feedback, Hobbys, soziale Interaktionen und Ziele) mit jeweils drei bis vier Items zu sechs PVS-Subkonstrukten (initiale Ansprechbarkeit auf Belohnung, Belohnungserwartung, Belohnungsantizipation, Belohnungssättigung, Belohnungsbewertung, Anstrengungsbewertung). Die PVSS-21 zeigte in drei verschiedenen Stichproben eine hohe interne Konsistenz, Test-Retest-Reliabilität und faktorielle Stabilität sowie eine gute externe Validität gemessen an etablierten Fragebögen. Ein hierarchisches Faktorenmodell erklärte dabei die Daten am besten: Sieben Faktoren auf erster Ebene, gemäß den sieben Belohnungstypen, bildeten einen starken übergeordneten generellen Faktor (Belohnungserleben insgesamt). Aber auch die Subskalen (Belohnungstypen) waren intern konsistent und reliabel, sodass sie für bestimmte Forschungsfragen auch unabhängig vom Gesamtfragebogen verwendet werden können. Im Bereich der klinischen Validierung unterschied die PVSS-21 depressive von nichtdepressiven Individuen und sagte die Schwere der Anhedonie (auch bei Kontrolle für den Depressionsstatus) vorher. Hobbys waren die stärksten Prädiktoren für das klinische Outcome und differenzierten am besten zwischen depressiven und nichtdepressiven Individuen.

Mit der PVSS-21 steht damit erstmals ein umfassender PVS-RDoC-inspirierter, validierter Fragebogen für die Forschung zur Verfügung. Allerdings zeigen die Studien, dass die einzelnen PVS-Subkonstrukte im subjektiven Selbstbericht durch den Fragebogen nicht differenzierbar waren, in Übereinstimmung mit anderen Fragebogenstudien, etwa im Bereich der Anhedonie, die ebenfalls nur Belohnungstypen unterscheiden konnten (z. B. bei der DARS [31, 44]). Was bedeutet das? Sind sie damit weniger real? Es spricht zunächst lediglich dafür, dass neurobiologische Differenzierungen der PVS dem Selbsterleben, gemessen anhand von Fragebögen, offenbar nicht zugänglich sind. Es könnte jedoch sein, dass es PVS-Subtypen gibt, bei denen eher die Erwartung von Belohnung, bei anderen der Genuss von Belohnung und bei wieder anderen eher die Berechnungen der nötigen Anstrengung zum Erhalt der Belohnung gestört sind. Dies lässt sich aber nicht per Fragebogen herausfinden, wäre allerdings über andere Untersuchungsebenen möglich, wie behaviorale Paradigmen oder die funktionelle Bildgebung: Genau deshalb verfolgt das $\mathrm{RDoC}$ ja auch einen multidisziplinären Mehrebenenansatz. In der Bildgebung des Belohnungssystems gibt es dazu schon länger eine Reihe von Untersuchungen, die etwa die Antizipation verschieden wahrscheinlicher monetärer Belohnungen mit dem Vorhersagefehler gleichzeitig untersuchen (für eine frühe Arbeit vgl. [1]) oder die Belohnungstypen vergleichen [35]. Es existieren aber noch keine systematischen Untersuchungen zur neurobiologischen Differenzierung multipler oder gar aller PVS-Konstrukte oder Belohnungstypen. Zudem ist der Mehrebenenansatz mit der Schwierigkeit konfrontiert, dass die verschiedenen Ebenen auch unterschiedliche Schwerpunkte setzen. So erwies sich vor allem der neurobiologisch beim Menschen praktisch noch gar nicht untersuchte Belohnungstyp „Hobbys“ im Selbstbericht als prädiktiv für Psychopa- thologie, während etwa in der Bildgebung überwiegend mit dem sehr gut skalierbaren und leicht einsetzbaren sekundären Verstärker kleiner Geldmengen gearbeitet wird. Auch folgt die PVSS-21 dem RDoC-Ansatz darin, Belohnungslernen aus dem Selbstbericht komplett auszuschließen, obwohl dem Lernen aber möglicherweise gerade in Krisenzeiten wie der gegenwärtigen COVID-19(„,corona virus disease 2019“)-Pandemie eine wichtige Rolle bei der Stressbewältigung zukommt [9]. Und letztlich sei auch hier noch einmal darauf hingewiesen, dass im PVSS-21 wie bei fast allen anderen Fragebögen zur Belohnung (und zu Persönlichkeitseigenschaften) der biologisch und psychologisch so wichtige primäre Verstärker und Belohnungstyp Sexualität nicht berücksichtigt bzw. explizit ausgeschlossen wurde. In diesem Fall lag es einfach nur daran, dass das Item zu viel Varianz aufwies und nicht reliabel genug war. Das ist v. a. daher schade, da in anderen Untersuchungen, insbesondere in den USA, das Thema Sexualität auch aus kulturellen Gründen (Scheu, solche Fragen zu stellen oder zu beantworten) oft gar nicht untersucht wird. Während Fragebogenentwicklung oft auf möglichst kontext- und altersgruppenübergreifende Anwendbarkeit ihrer Fragen zielt, haben experimentelle Paradigmen den Vorteil, den tatsächlich erlebten Belohnungstyp kontrollieren zu können - natürlich mit der Einschränkung von Durchführbarkeit im Labor.

\section{Selbstbericht über PVS: Erfassung im Alltag durch mobile Geräte}

Eine inzwischen breit angewendete, alternative Methode zur Erfassung von Psychopathologie ist die Verwendung mobiler Geräte mit Apps, auch bekannt als EMA („ecological momentary assessment“), ESM („experience sampling methodology") oder digitale Phänotypisierung. Der Vorteil dieser Art der Datenerfassung liegt auf der Hand: Sie beschränkt sich nicht auf das Ausfüllen eines Fragebogens, bei dem man Auskunft über die letzten zwei Wochen geben muss mit allen möglichen Verzerrungen und Fehlern, sondern kann das momentane Befinden mehrmals 


\begin{tabular}{|c|c|}
\hline $\begin{array}{l}\text { Der Patient wird darüber } \\
\text { informiert, dass mit der } \\
\text { Befragung etwas über seine } \\
\text { früheren und gegenwärtigen } \\
\text { Motivationen, Werte, Ziele, } \\
\text { Belohnungen, Verhaltens- }\end{array}$ & $\begin{array}{l}\text { Zu Motivationen, Werten und Anstrengung, z.B. } \\
\text { - Was motiviert, inspiriert und treibt Sie an? Wofür brennen Sie? Wonach streben Sie? } \\
\text { - Welche Werte sind Ihnen wichtig? Welche sind relevant für Ihre Selbstachtung? } \\
\text { - Wie weit in die Zukunft reichen Ihre Pläne, Vorstellungen und Wünsche? } \\
\text { - Welche Ihrer Motivationen sehen Sie als positiv an? Welche als potenziell negativ oder schädlich für Sie selbst? } \\
\text { - Wie energisch und beharrlich sind Sie bei der Verfolgung Ihrer Ziele? Bei welchen Zielen geben Sie zu leicht auf? } \\
\text { - Wie gut sind Sie darin, Ihre Strategien zur Erreichung ihrer Ziele zu ändern, wenn die Umstände es erfordern, z.B. } \\
\text { indem Sie andere Wege einschlagen oder ein alternatives Ziel verfolgen? Wie flexibel sind Sie dabei? }\end{array}$ \\
\hline $\begin{array}{l}\text { auslöser, Beharrlichkeit, } \\
\text { Befriedigungen, Impulse, } \\
\text { Abhängigkeiten und } \\
\text { Gewohnheiten herausge- } \\
\text { funden werden soll. } \\
\text { Die Fragen können so gestellt } \\
\text { werden, dass der Befragte sie aus }\end{array}$ & $\begin{array}{l}\text { Zu Entscheidungen und Bewertungen, z.B. } \\
\text { - Wie würden Sie Ihre Urteilsfähigkeit beschreiben, abzuschätzen, was in Schule, Arbeit, Beziehungen und anderen } \\
\text { Aktivitäten realistisch erreichbar ist? } \\
\text { - Worauf gründen Ihre Entscheidungen? Vernunft und Logik (Listen mit Pro und Kontra machen)? Gefühlen und } \\
\text { Emotionen (wie eine Entscheidung Ihre Gefühle beeinflusst)? Impulsivität (reagieren Sie aus dem Moment heraus)? } \\
\text { - Wie stark tendieren Sie dazu, unrealistisch optimistisch, idealistisch oder pessimistisch zu sein? } \\
\text { - Inwiefern sind Sie dazu in der Lage kluge, „weise“ Handlungspläne zu entwerfen und umzusetzen? Wie häufig treffen } \\
\text { Sie unkluge Entscheidungen in wichtigen Angelegenheiten? }\end{array}$ \\
\hline $\begin{array}{l}\text { eigener Perspektive oder aus der } \\
\text { einer ihm nahestehenden Person } \\
\text { beantworten soll. }\end{array}$ & $\begin{array}{l}\text { Zu Abhängigkeiten, Impulsen und Gewohnheiten, z.B. } \\
\text { - Auf welche Substanzen, Dinge oder Aktivitäten sind Sie zu stark angewiesen oder gar davon abhängig? } \\
\text { - Bei welcher Art von Impulsen müssen Sie sich anstrengen, um ihnen nicht nachzugeben? Wie gut oder weniger gut } \\
\text { gelingt Ihnen das? Welche Konsequenzen ergeben sich, wenn Sie Ihren Impulsen nachgeben? } \\
\text { - In welcher Hinsicht sind Sie ein Gewohnheitstier? Was würden Sie als gute/schlechte/schädliche Gewohnheiten } \\
\text { bezeichnen? Was machen Ihre Gewohnheiten mit Ihnen? Was ist der Preis, den Sie für Ihre Gewohnheiten zahlen? }\end{array}$ \\
\hline
\end{tabular}

Abb. 2 \ Durch positive Valenzsysteme der Research-Domain-Criteria inspirierte klinische Evaluation. (Mod. nach [45])

täglich im normalen Lebenskontext der Personen einfach erfassen und messen. Natürlich lässt sich dies auch für die Erfassung von RDoC-Konstrukten benutzen und damit dessen Potenzial erweitern, wie Torous et al. [37] für das $\mathrm{RDoC}$ insgesamt überzeugend argumentiert haben. Torous et al. weisen insbesondere darauf hin, dass die digitale Phänotypisierung gerade für die Kombination und Integration verschiedener Analyseebenen besonders geeignet ist, beispielsweise in der kombinierten Messung von Selbstbericht, physiologischen Maßen (Puls, Hautleitfähigkeit), Bewegungsdaten (GPS) und sozialen Interaktionen (z. B. Chat-Verläufe). Einige neuere Bildgebungsstudien, gerade auch im Bereich der PVS, versuchen, neuronale Aktivitäten während klassischer experimenteller Belohnungsparadigmen mit entsprechendem Verhalten und Erleben im Lebensalltag der Personen in Verbindung zu bringen. In der Studie von Bakker et al. [3] korrelierte beispielsweise der neuronale Belohnungsvorhersagefehler während eines probabilistischen Lernexperimentes mit dem Zusammenhang von Belohnungsantizipation und später tatsächlich erlebter Belohnungsantwort im echten Leben, gemessen mittels Smartphone-App in einer Stichprobe von jungen leicht bis moderat depressiven Probanden ohne Medikation. Auch der zeitliche Verlauf positiver emotionaler Reaktionen bei anhedonen Personen lässt sich durch ESM detailliert untersuchen (vgl. z. B. [15, 16]). Für einen Überblick zur Verwendung von Selbstbericht im Alltag durch ESM zur Erforschung mentaler Gesundheit siehe Myin-Germeys et al. [26].

In Bezug auf die Methoden der digitalen Phänotypisierung wird es von besonderem Interesse sein, wenn erste Übersichten und Metaanalysen erscheinen, die systematisch zu erfassen suchen, welche der neun Subkonstrukte der PVS schon untersucht worden sind und vor allem, ob durch diese Methoden andere Ergebnisse gefunden werden als durch traditionellere Untersuchungen.

\section{Klinische Brauchbarkeit: RDoC-PVS im klinischen Alltag}

Eine anhaltende Kritik am RDoC ist seine angeblich geringe klinische Relevanz. Doch auch hierzu gibt es neue, innovative Ansätze, z. B. wie man DSM-5 und $\mathrm{RDoC}$ in einem hybriden Ansatz verbinden kann. Einen detailliert ausgearbeiteten Vorschlag bietet etwa die folgende Arbeit von Yager et al., deren Titel Programm ist: „Potenzielle Anwendung der Research Domain Criteria (RDoC) des NIMH auf die klinische Praxis: Wie $\mathrm{RDoC}$ in Untersuchung, diagnostischen Prozessen, Fallformulierung, Behandlungsplanung und klinischen Notizen verwendet werden könnte“ ([45]; Übersetzung H.W.). Die Autoren entwerfen für alle (damals fünf) Domänen der RDoC-Matrix klinische Vorschläge, indem sie die RDoC-Konstrukte klinisch adaptieren und konkret darstellen, wie sie eingesetzt werden können. Im Bereich der PVS etwa schlagen sie vor, dass PVS aus klinischer Sicht vor allem mit Antrieb und Anstrengungsallokation zu tun haben. Sie benennen drei Subkategorien, die sie mit PVS-Konstrukten begründen: (1) Motivation, Werte, Anstrengung, (2) Entscheidungsfindung und Bewertung sowie (3) Abhängigkeiten, Impulse und Gewohnheiten. Eine strukturierte Abfrage dieser Aspekte erlaubt, ein klinisch reichhaltigeres Bild des Patienten zu entwickeln und den Patienten klinisch systematisch und genauer zu explorieren (vgl. • Abb. 2).

Anschließend zeigen sie beispielhaft am Fall eines 55-jährigen Patienten, der die DSM-5-Diagnose einer rezidivierenden Depression, einer posttraumatischen Belastungsstörung (nach Verkehrsunfall) und einer Alkoholgebrauchsstörung erfüllt, wie klinisch relevante Informationen zu den fünf Domänen in die Fallformulierung, Behandlungsplanung und klinischen Notizen integriert werden können (für Details vgl. [45]). 


\section{Schlussfolgerungen}

Anhand der PVS lässt sich in mehrerer Hinsicht die Idee des RDoC gut demonstrieren. Es handelt sich um eine neurokognitive Domäne, die sowohl beim Tier als auch beim Menschen gut untersucht ist. Dies erklärt, warum die Subkonstrukte aufgenommen (bekannte neuronale Schaltkreise) und warum sie über die Zeit geändert, neu arrangiert und ergänzt wurden (neue Erkenntnisse, Revisionsfähigkeit).

Einige Vorteile des RDoC-Ansatzes sind deutlich geworden. Im Bereich der PVS zeigt sich, dass klinisch relevante, diagnoseunabhängige Symptome damit sehr gut erklärt bzw. besser verstanden werden können (Anhedonie). Die Revision der PVS-Matrix illustriert, wie RDoC-Konstrukte dem aktuellen, gesicherten Forschungsstand angepasst werden. Gleichzeitig beeinflusst RDoC wiederum die Erforschung der PVS. Die Beispiele Ess- und Autismusspektrumstörungen machen deutlich, inwiefern die RDoC-Matrix neue Modellbildungen und systematische Untersuchungen ermöglicht. Zudem gibt die RDoC-Initiative auch konkrete Hinweise, welche Messinstrumente auf den verschiedenen Ebenen der Analyse eingesetzt werden sollten, um ein Konstrukt verlässlich zu erfassen. Der Fokus auf Selbstbericht und klinische Nützlichkeit zeigt, dass RDoC über die reine Wissenschaft hinaus neue klinische Differenzierungen ermöglicht und von methodischen Weiterentwicklungen (Beispiel digitale Phänotypisierung) profitiert.

Gleichzeitig werden auch Limitationen des RDoC deutlich, die sich auf die klinische Verwendbarkeit auswirken. Es demonstriert konzeptuelle Unklarheiten (Anhedonie wird offiziell - noch - unter „loss“ gelistet und findet sich als soziale Anhedonie auch in der Domäne „soziale Systeme“). Es fehlt ein Bezug bzw. der Einbezug einer differenzierten Psychopathologie. Es gibt kein gutes Konzept, wie man die verschiedenen Ebenen der Untersuchungen integrieren kann, zumal die Differenzierbarkeit der Konstrukte je nach Ebene variieren kann, oder wie man damit umgeht, wenn sich zwischen Ebenen zu schwache Bezüge finden. Weiter- hin zeigt sich, dass die Beschränkung auf neuronale Schaltkreise viele Phänomene auslässt. Im Bereich positiver Emotionen gibt es eine reichhaltige Literatur zu differenzierten positiven Erfahrungen, Einstellungen und Konzepten, die insbesondere im menschlichen Bereich eine Rolle spielen. Ein Teil davon findet sich in der Domäne „soziale Systeme“ wie etwa Zugehörigkeit, Bindung oder Empathie, andere Teile werden aber (noch) nicht berücksichtigt wie etwa Zufriedenheit, Verliebtheit, Hoffnung, Stolz, Dankbarkeit oder Ehrfurcht. Sogenannte eudaimonische Zustände oder Erlebnisse wie etwa Authentizität, Sinn, Exzellenz und (posttraumatisches) Wachstum [33, 42], die Themen der positiven Psychologie sind, finden ebenfalls keine Berücksichtigung, was aber aufgrund der (noch) mangelnden neurobiologischen Befunde wenig verwundert.

Wie sieht die Zukunft der Entwicklung der PVS aus? Zum einen bietet die PVS-Matrix schon jetzt einen guten Rahmen für das systematische Erheben von Daten und die Entwicklung von Krankheitsmodellen. Dies gilt sowohl für DSM-5-definierte Spektrumserkrankungen als auch für transdiagnostische Symptome und Syndrome. Die positiven Valenzen bieten eine hochinteressante Verbindung von eher kognitiver Neurowissenschaft und molekularen Ansätzen, da viele Signalstoffe im Bereich positiver Erfahrungen von Bedeutung sind (Dopamin, endogene Opioide, Serotonin, Neuropeptide wie Oxytocin und Vasopressin u. a.). Eine interessante Herausforderung bleiben Ansätze, die nicht nur transdiagnostisch, sondern auch domänenübergreifend zwischen PVS und den (Sub)Konstrukten von Zugehörigkeit und Bindung im Bereich der sozialen Systeme Verbindungen herstellen. Dies gilt insbesondere für soziale positive Konstrukte im Bereich der Sexualität und der Paarbindung. Insgesamt ist zu hoffen, dass zukünftige Revisionen der RDoC-Matrix die Entwicklungen im Bereich der positiven Psychologie integrieren werden.

\section{Fazit für die Praxis}

\section{- Die positiven Valenzsysteme (PVS) des RDoC (Research-Domain-Criteria) beziehen sich alle auf Facetten der dopaminvermittelten Belohnungs- verarbeitung. \\ - Die PVS-Matrix ist hilfreich für die systematische Erforschung von Störungen, bei denen die Beloh- nungsverarbeitung bisher nicht nicht im Vordergrund stand (Essstörungen, Autismus) sowie für transdiagnosti- sche Symptome wie die Anhedonie. \\ - Es wurden validierte Skalen zur Erfassung der PVS-Konstrukte der Belohnungsverarbeitung entwickelt. \\ - Zukünftige Forschungen zu den PVS sollten über die Belohnungspro- zesse hinaus auch andere positive Konstrukte und die Rolle weite- rer molekularer Vermittler stärker berücksichtigen und integrieren.}

\section{Korrespondenzadresse}

\section{Prof. Dr. Dr. Henrik Walter}

Klinik für Psychiatrie und Psychotherapie CCM, Forschungsbereich Mind and Brain, Charité Universitätsmedizin Berlin, corporate member of Freie Universität Berlin and HumboldtUniversität zu Berlin

Charitéplatz 1, 10117 Berlin, Deutschland henrik.walter@charite.de

Förderung. Die Arbeit von A.D. und S.A.W. wird gefördert durch die Deutsche Forschungsgemeinschaft (DFG) - 337619223/GRK2386.

\section{Einhaltung ethischer Richtlinien}

Interessenkonflikt. H. Walter, A. Daniels und S.A. Wellan geben an, dass kein Interessenkonflikt besteht.

Für diesen Beitrag wurden von den Autoren keine Studien an Menschen oder Tieren durchgeführt. Für die aufgeführten Studien gelten die jeweils dort angegebenen ethischen Richtlinien.

\section{Literatur}

1. Abler B, Walter H, Erk S et al (2006) Prediction error as a linear function of reward probability is coded in human nucleus accumbens. Neuroimage 31:790-795

2. American Psychiatric Association (2013) Diagnostic and statistical manual of mental disorders. American Psychiatric Association, Washington, DC

3. Bakker JM, Goossens L, Kumar P et al (2019) From laboratory to life: associating brain reward 
processing with real-life motivated behaviour and symptoms of depression in non-help-seeking young adults. Psychol Med 49:2441-2451

4. Berridge KC, Kringelbach ML (2015) Pleasure systems in the brain. Neuron 86:646-664

5. Berwian IM, Wenzel JG, Collins AGE et al (2020) Computational mechanisms of effort and reward decisions in patients with depression and their association with relapse after antidepressant discontinuation. JAMA Psychiatry 77:513-522

6. Clark L, Boileau I, Zack M (2019) Neuroimaging of reward mechanisms in gambling disorder: an integrative review. Mol Psychiatry 24:674-693

7. Corlett PR, Murray GK, Honey GD et al (2007) Disrupted prediction-error signal in psychosis: evidence for an associative account of delusions. Brain 130:2387-2400

8. Cuthbert BN, Insel TR (2013) Toward the future of psychiatric diagnosis: the seven pillars of $\mathrm{RDoC}$. BMCMed 11:126

9. Daniels A, Wellan SA, Walter H (2021) Learning it the hard way-how enjoying life and positive appraisal buffer the negative effects of stressors on mental health in the COVID-19 pandemic. J Affect Disord Rep. https://doi.org/10.1016/j.jadr.2021. 100200

10. Der-Avakian A, Markou A (2012) The neurobiology of anhedonia and other reward-related deficits. Trends Neurosci 35:68-77

11. Ermakova AO, Knolle F, Justicia A et al (2018) Abnormal reward prediction-error signalling in antipsychotic naive individuals with firstepisode psychosis or clinical risk for psychosis. Neuropsychopharmacology 43:1691-1699

12. Everitt BJ, Robbins TW (2005) Neural systems of reinforcement for drug addiction: from actions to habits to compulsion. Nat Neurosci 8:1481-1489

13. Gard DE, Gard MG, Kring AM et al (2006) Anticipatory and consummatory components of the experience of pleasure: a scale development study. J Res Pers 40:1086-1102

14. Hallford DJ, Sharma MK (2019) Anticipatory pleasure for future experiences in schizophrenia spectrum disorders and major depression: a systematic review and meta-analysis. $\mathrm{Br} \mathrm{J}$ Clin Psychol 58:357-383

15. Heininga VE, Dejonckheere $E$, Houben $M$ et al (2019) The dynamical signature of anhedonia in major depressive disorder: positive emotion dynamics, reactivity, and recovery. BMCPsychiatry 19:59

16. Heininga VE, van Roekel $E$, Ahles JJ et al (2017) Positive affective functioning in anhedonic individuals' dailylife: anything but flat and blunted. JAffect Disord 218:437-445

17. Heinz A, Beck A, Halil MG et al (2019) Addiction as learned behavior patterns. J Clin Med 8:1086

18. Husain M, Roiser JP (2018) Neuroscience of apathy and anhedonia: a transdiagnostic approach. Nat Rev Neurosci 19:470-484

19. Insel T (2013) Transforming diagnosis. https:// www.nimh.nih.gov/about/directors/thomasinsel/blog/2013/transforming-diagnosis.shtml. Zugegriffen: 12.Apr. 2021

20. Jacobson NS, Martell CR, Dimidjian S (2001) Behavioral activation treatment for depression: returning to contextual roots. Clin Psychol 8:255-270

21. Keren H, O'callaghan G, Vidal-Ribas P et al (2018) Reward processing in depression: a conceptual and meta-analytic review across $\mathrm{FMRI}$ and EEG studies. Am J Psychiatry 175:1111-1120
22. Khazanov GK, Ruscio AM, Forbes CN (2020) The positive valence systems scale: development and validation. Assessment 27:1045-1069

23. Kohls G, Yerys BE, Schultz RT (2014) Striatal development in autism: repetitive behaviors and the reward circuitry. Biol Psychiatry 76:358-359

24. Lambert C, Da Silva S, Ceniti AK et al (2018) Anhedonia in depression and schizophrenia: a transdiagnostic challenge. CNS Neurosci Ther 24:615-623

25. Lewinsohn PM, Sullivan JM, Grosscup SJ (1980) Changing reinforcing events: an approach to the treatment of depression. Psychotherapy 17:322-334

26. Myin-Germeys I, Kasanova Z, Vaessen T et al (2018) Experience sampling methodology in mental health research: new insights and technical developments. World Psychiatry 17:123-132

27. National Institute of Mental Health (2011) Positive valence systems: workshop proceedings. https:// www.nimh.nih.gov/research/research-fundedby-nimh/rdoc/positive-valence-systemsworkshop-proceedings.shtml. Zugegriffen: 12. Apr. 2021

28. National Institute of Mental Health (2018) RDoC changes to the matrix (CMAT) workgroup update: addition of the sensorimotor domain. https:// www.nimh.nih.gov/about/advisory-boards-and groups/namhc/reports/rdoc-changes-to-thematrix-cmat-workgroup-update-addition-ofthe-sensorimotor-domain.shtml. Zugegriffen: 12. Apr. 2021

29. National Institute of Mental Health (2018) $\mathrm{RDoC}$ changes to the matrix (CMAT) workgroup update: proposed positive valence domain revisions. https://www.nimh.nih.gov/about/advisoryboards-and-groups/namhc/reports/cmat-pvsreport-508_157003.pdf. Zugegriffen: 12. Apr 2021

30. Ribot T (1896) La psychologie des sentiments. Felix Alcan, Paris

31. Rizvi SJ, Quilty LC, Sproule BA et al (2015) Development and validation of the dimensional anhedonia rating scale (DARS) in a community sample and individuals with major depression. Psychiatry Res 229:109-119

32. Schaefer LM, Steinglass JE (2021) Reward learning through the lens of RDoC: a review of theory, assessment, and empirical findings in the eating disorders. Curr Psychiatry Rep 23:2

33. Schnell T, Krampe H (2020) Meaning in life and self-control buffer stress in times of COVID-19: moderating and mediating effects with regard to mental distress. Front Psychiatry 11:582352. https://doi.org/10.3389/fpsyt.2020.582352

34. Schultz W (2011) Potential vulnerabilities of neuronal reward, risk, and decision mechanisms to addictive drugs. Neuron 69:603-617

35. Sescousse G, Caldú X, Segura B et al (2013) Processing of primary and secondary rewards: a quantitative meta-analysis and review of human functional neuroimaging studies. Neurosci Biobehav Rev 37:681-696

36. Starr LR, Hershenberg R (2017) Depressive symptoms and the anticipation and experience of uplifting events in everyday life. J Clin Psychol 73:1442-1461

37. Torous J, Onnela JP, Keshavan M (2017) New dimensions and new tools to realize the potential of RDoC: digital phenotyping via smartphones and connected devices. TransI Psychiatry 7:e1053

38. Treadway MT, Bossaller NA, Shelton RC et al (2012) Effort-based decision-making in major depressive disorder: a translational model of motivational anhedonia. J Abnorm Psychol 121:553-558

39. Trøstheim M, Eikemo M, Meir R et al (2020) Assessment of anhedonia in adults with and without mental illness: a systematic review and meta-analysis. JAMA Netw Open 3:e2013233

40. Tschida JE, Yerys BE (2021) A systematic review of the positive valence system in autism spectrum disorder. Neuropsychol Rev 31:58-88

41. Walter $H$ (2017) Research domain criteria (RDoC): psychiatric research as applied cognitive neuroscience. Nervenarzt 88:538-548

42. Walter H, Wellan SA, Daniels A (2020) Glück im Unglück. (An)hedonie aus Sicht der kognitiven Neurowissenschaft und Psychiatrie. In: Fink $\mathrm{H}$, Rosenzweig R (Hrsg) Hirn im Glück. Freude, Liebe, Hoffnung im Spiegel der Neurowissenschaft. Kortizes, Nürnberg, S67-90

43. Wang S, Leri F, Rizvi SJ (2021) Anhedonia as a central factor in depression: neural mechanisms revealed from preclinical to clinical evidence. Prog Neuropsychopharmacol Biol Psychiatry 110:110289

44. Wellan SA, Daniels A, Walter H (2021) State anhedonia in young healthy adults: psychometric properties of the German dimensional anhedonia rating scale (DARS) and effects of the COVID-19 pandemic. Front Psychol. https://doi.org/10.3389/ fpsyg.2021.682824

45. Yager J, Feinstein RE (2017) Potential applications of the national institute of mental health's research domain criteria (RDoC) to clinical psychiatric practice: how RDoC might be used in assessment, diagnostic processes, case formulation, treatment planning, and clinical notes. J Clin Psychiatry 78:423-432 